\title{
Modelagem de Construções Interrogativas QU- no Constructicon da FrameNet Brasil
}

\author{
Natália Duarte Marção' ${ }^{1}$ Tiago Timponi Torrent ${ }^{1}$ \\ ${ }^{1}$ FrameNet Brasil - Programa de Pós-Graduação em Linguística \\ Universidade Federal de Juiz de Fora (UFJF) \\ Rua José Lourenço Kelmer, $\mathrm{s} / \mathrm{n}^{\circ}$, Campus Universitário \\ 36036-900 - Juiz de Fora - Minas Gerais - Brasil \\ natalia.duartedestudante.ufjf.br, tiago.torrenteufjf.edu.br
}

\begin{abstract}
This paper aims to present the description and the linguisticcomputational modeling of Wh-interrogative constructions in Brazilian Portuguese in the FrameNet Brasil Constructicon.
\end{abstract}

Resumo. Este trabalho objetiva apresentar a descrição e a modelagem linguístico-computacional das construções interrogativas $Q U$-do Português brasileiro no Constructicon da FrameNet Brasil.

\section{Introdução}

O presente trabalho tem por objetivo apresentar a descrição e a modelagem das construções Interrogativas QU- do Português Brasileiro como resultado da pesquisa realizada por Marção (2018) durante o mestrado. Para o Português do Brasil, existem algumas análises para as construções Interrogativas QU- sob abordagens diversas. Dentre essas abordagens, destacamos as gerativistas [Adger 2003; Augusto 2005; Modesto 2012], as quais propõem que o fenômeno sintático de maior interesse de análise se relaciona ao movimento do elemento QU- em construções interrogativas em Português Brasileiro, uma vez que existe a possibilidade do movimento, mas este não é obrigatório como ocorre em outras línguas, por exemplo, o inglês.

De acordo com as abordagens gerativistas [Adger 2003; Augusto 2005; Modesto 2012], ao mover o elemento QU- para a periferia à esquerda da sentença, este deixa uma cópia em sua posição de origem, a qual não é realizada foneticamente, mas é interpretada semanticamente nesta posição. Dessa maneira, o elemento movido é realizado foneticamente, enquanto a cópia é apagada no componente fonológico. Em contrapartida, a cópia é interpretada semanticamente em sua posição de origem e não na posição ocupada após o movimento.

Focar no movimento do elemento QU- nas construções interrogativas não é suficiente para abranger todas as especificidades desse tipo de construção. Dessa forma, para nosso trabalho, as abordagens construcionistas se mostram mais adequadas, conforme se mostrará na seção que se segue.

\section{Proposta Construcionista para as Construções Interrogativas QU-}

Neste trabalho, recorremos à Gramática das Construções de Berkeley (Berkeley Construction Grammar - BCG) [Kay \& Fillmore 1999; Fillmore 2013] e à Gramática das Construções Baseada em Signos (Sign-Based Contruction Grammar - SBCG) [Sag 
2012] pelo seu caráter formal, visto que se tem por objetivo descrever e modelar construções em termos de suas propriedades gramaticais e seu potencial semântico.

Essas bases teóricas propõem que as informações sintático-semânticas de uma construção podem ser representadas através de um sistema de traços em Matrizes de Atributo e Valor (AVM). A AVM é a forma em que geralmente são apresentadas as estruturas de traços, assim, as estruturas de traços consistem na "divisão" de um dado linguístico em partes menores, os chamados atributos e a cada atributo associam-se valores.

De maneira similar, a SBCG busca formalizar descrições que sejam interpretáveis computacionalmente, integrando-as aos pressupostos fundamentais das abordagens construcionistas. Na SBCG, o pareamento de forma e significado é mediado por signos. Sag (2012) destaca que descrição de um signo incorpora traços como fonologia, forma, estrutura argumental, sintaxe, semântica e contexto e esses traços são descritos também em termos de AVMs, assim como proposto pela BCG.

Uma das principais características das estruturas de traços é poderem combinar informações através da unificação. O processo de unificação é o responsável pela aceitação ou rejeição de constituintes candidatos a ocupar posições sintáticas específicas. Portanto, a aceitação ou restrição fica condicionada à compatibilidade entre os valores dos atributos, isto é, aos elementos linguísticos e os valores exigidos pelas posições da construção. Assim, é possível definir e especificar os tipos de entidades que podem ou devem estar em cada sintagma [Fillmore 2013].

É através do processo de unificação que as AVMs combinam-se e, assim, projetam uma nova AVM que contém exatamente os valores e atributos das AVMs que se uniram [Goldberg 2006]. Logo, a principal tarefa da unificação é garantir que os atributos que possuem valores contraditórios falhem ao se combinar, de modo a não chegar a licenciar uma construção [Fried \& Ostman 2004].

Sob a perspectiva da BCG, nas construções interrogativas, o elemento no início da sentença na posição mais periférica à esquerda, mantém uma ligação via unificação com uma lacuna de tal forma que, no lugar da lacuna, se mantêm as propriedades do constituinte de modo a tornar a sentença interpretável [Fillmore 1985]. Fillmore (1985) explica que os estudos gerativistas propõem que o constituinte QU- é movido para a posição inicial da sentença, além de carregar as propriedades necessárias para que ele seja interpretado na posição de origem. Ao assumir essa nova posição, esse constituinte também adquire as características exigidas por sua nova posição. Já os estudos construcionistas, segundo Fillmore (1985), se concentram nas características da posição na qual o constituinte QU- de fato ocorre.

Já a SBCG desenvolve mais a abordagem construcionista para as interrogativas QU-, que são entendidas, nesse modelo, como um tipo de construção filler-gap, isso porque o sintagma QU- preenche a posição inicial da sentença enquanto é deixada uma lacuna na posição em que o sintagma é interpretado. Sobre a dependência existente entre o constituinte inicial e a lacuna deixada por este, Sag (2012) afirma que pode haver uma discrepância entre os atributos e seus valores que surge da realização nãolocal do constituinte. De modo a formalizar essa discrepância, o autor propõe que

a presença de uma lacuna é codificada em termos de uma especificação não vazia para o traço GAP (por exemplo, $[\mathrm{GAP}<\mathrm{NP}>]$ ). Por contraste, uma 
expressão que não contenha lacunas desvinculadas é especificada como [GAP <>] [SAG 2012:163, tradução nossa].

Nas construções do tipo filler-gap, Sag (2012), argumenta que ocorre um processo de Percolação. Nesse processo, as informações de contextos sintáticos mais baixos são passadas para contextos sintáticos superiores por restrições, logo, quando todas as AVMs filhas têm uma especificação vazia para um determinado traço, 0 mesmo ocorrerá com a AVM mãe. Contudo, quando uma das filhas tem uma especificação não-vazia, a mãe, então, vai carregar a mesma especificação não-vazia. Essa questão é formalizada no diagrama apresentado na Figura 1.

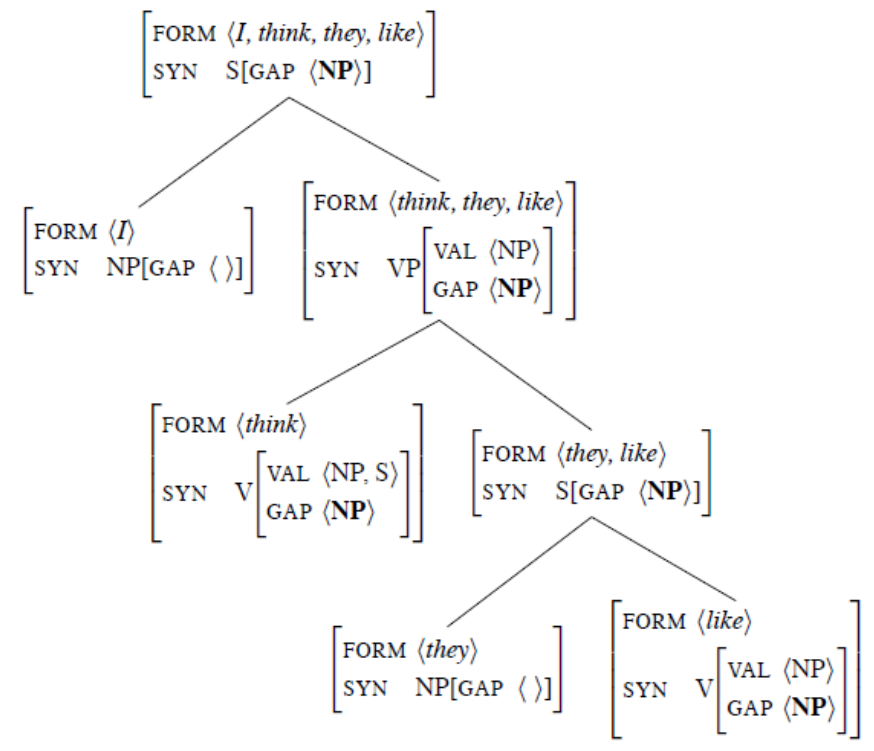

Figura 1. Representação do processo de Percolação [Sag 2012:163]

Na Figura 1, podemos ver que a especificação não-vazia para o traço GAP ([GAP $<\mathrm{NP}>]$ ) foi "filtrada" pelas filhas e passada aos níveis superiores. Diante disso, Sag (2012) afirma que para o traço QU (WH) também ocorre o processo de Percolação. Assim, o traço QU tem um valor não-vazio que percola os níveis mais baixos das AVMs até atingir os níveis superiores.

A partir do que foi exposto, podemos dizer que a SBCG fornece uma abordagem ao tratamento das construções Interrogativas QU-, que, principalmente pela formalização proposta pelas AVMs, contribui para o tratamento computacional destas construções.

\section{Constructicon da FrameNet Brasil}

A partir dos pressupostos da BCG e da SBCG, o Constructicon pode ser definido como um recurso computacional sintático-semântico que contém o repertório das construções de uma língua [Fillmore 2008]. O Constructicon tem como propósito suprir a necessidade de análise de estruturas linguísticas que não são processáveis lexicograficamente, visto que, numa FrameNet, somente as valências das unidades lexicais são anotadas. O Constructicon, mais especificamente, descreve construções em termos de suas propriedades gramaticais e de seu potencial semântico. Ademais, realiza tais descrições de modo compatível com os pressupostos teóricos da BCG. 
Um dos desafios de desenvolver um Constructicon, de acordo com Torrent et al. (2018), é o de representar computacionalmente as restrições e possibilidades de construções, por exemplo, como os elementos da construção se relacionam com outra construção, ou como o polo formal se relaciona com o polo semântico. Logo, é necessário que o Constructicon abarque questões relacionadas a constituência das construções, bem como, o processo de unificação.

Sobre a constituência, Fillmore et al. (2012) discorrem que, no Constructicon, cada construção é definida em termos de suas partes constituintes, os chamados Elementos da Construção (EC). Dessa forma, o processo de modelagem das construções se dá, primeiramente, pela definição das propriedades sintáticas destas construções.

$\mathrm{Na}$ FrameNet Brasil, o Constructicon realiza esse processo de forma mais integrada do ponto de vista da base de dados, uma vez que permite associar padrões construcionais a frames, além de permitir a adição de restrições às construções modeladas [Torrent et al. 2018]. Para ilustrar como ocorre essa integração do banco de dados, tomamos como exemplo a construção Transitiva_indireta_agentiva. Ela é constituída pelos ECs Sujeito e Predicado. Para essa construção, no processo de unificação, podemos estabelecer restrições de constituência. Dessa forma, o EC Sujeito é licenciado pela construção Sintagma_nominal_determinado, enquanto o EC Predicado

é uma instância de um dos subtipos da construção Sintagma_verbal_com_complemento_preposicionado.

Ademais, o Constructicon permite estabelecer relações entre construção e frame, assim, uma construção como a Transitiva_indireta_agentiva evoca o frame de Ação_transitiva. Esse frame é basicamente definido como a ação de um Agente que afeta uma entidade, chamada de Paciente. Quando a construção evoca um frame, os ECs referem-se aos Elementos de Frame (EFs) do frame evocado pela construção. Os EFs são as partes constituintes de um frame. Sendo assim, os ECs Sujeito e Predicado da construção Transitiva_indireta_agentiva evocam, respectivamente, os EFs Agente e Paciente do frame Ação_transitiva.

As especificidades do Constructicon da FrameNet Brasil, serão mais desenvolvidas na próxima seção, na qual demonstraremos como é feita a inclusão das construções neste modelo linguístico-computacional.

\section{Modelagem das Construções Interrogativas QU- no Constructicon}

A modelagem de construções no Constructicon da $\mathrm{FN}-\mathrm{Br}$ segue algumas etapas, como propõe Marção (2018), e estas serão apresentadas aqui.

Segundo Marção (2018), o processo de modelagem das construções Interrogativas QU- se iniciou pela modelagem de duas construções abstratas, a Interrogativa_QU e a Interrogativa_QU_preposicionada, as quais são concebidas como nós abstratos em relação às demais construções interrogativas modeladas e não podem licenciar construtos na língua. Essas construções abstratas congregam informações que são compartilhadas por todas as construções filhas, ou seja, as construções que herdam dela, além de ter a função de organizar as demais construções em rede.

Para exemplificar o processo de modelagem, Marção (2018) utiliza a construção Interrogativa_qual para demonstrar os passos que devem ser seguidos com base no emprego da ferramenta WebTool 3.0 da FrameNet Brasil. 
O passo inicial da modelagem é definir os elementos que constituem a construção, i. e., os Elementos de Construção (ECs), assim sendo, a construção Interrogativa_qual é constituída pelos ECs Estrutura_argumental_base e Pronome_qual. Nesse contexto, para inserção da construção Interrogativa_qual, é necessário apontar três informações iniciais, como observado na Figura 2.

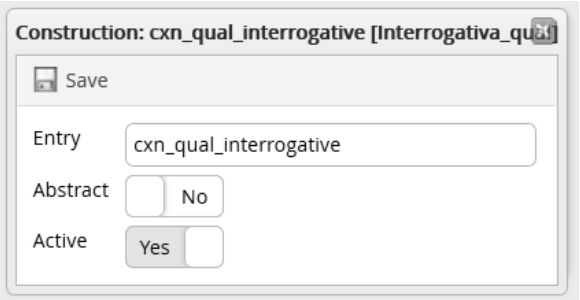

Figura 2. Edição de entrada da construção Interrogativa_qual [Marção 2018:82]

Em "Entry" é registrado o nome interlingual da construção. O nome é composto pela abreviação de construção "cnx" e o restante do nome é uma especificação da construção em questão (Interrogativa_qual). Em "abstract" sinalizamos se é uma construção abstrata ou não, no caso, está marcado "no", pois não se trata de uma construção abstrata. Por fim, em "Active" assinalamos se essa construção está ou não ativa na base de dados. Feito isso, o processo é salvo e em seguida uma nova tela se abre, a qual é apresentada na Figura 3.

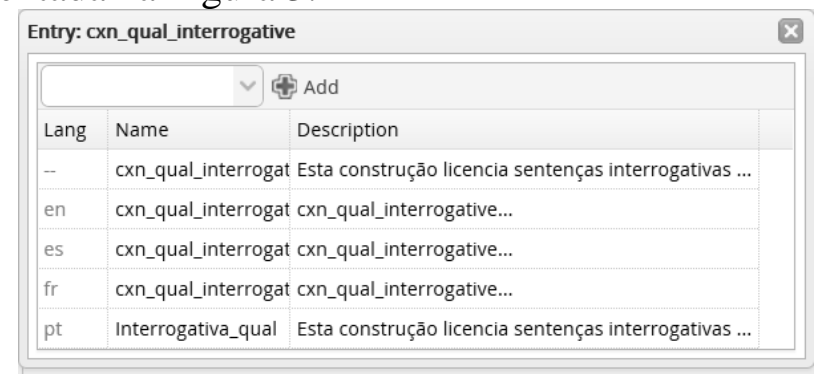

Figura 3. Edição de entrada por idioma [Marção 2018:82]

Ao clicar em alguma abreviação de idioma é possível editar a entrada de cada idioma de forma legível a humanos. Se clicarmos em " $\mathrm{pt}$ " somo redirecionados a outra tela (Figura 4).

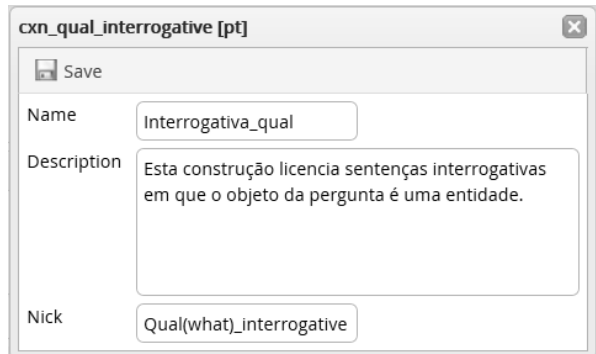

Figura 4. Edição da definição da construção em Português Brasileiro [Marção 2018:83]

Marção (2018) ressalta que na tela da Figura 4, é possível alterar o nome da construção e criar uma definição para ela em Português Brasileiro, contudo, em "Nick" é mantido o nome em inglês da construção. A partir disso, a construção foi criada e, logo, passa-se a criação e definição dos ECs. 


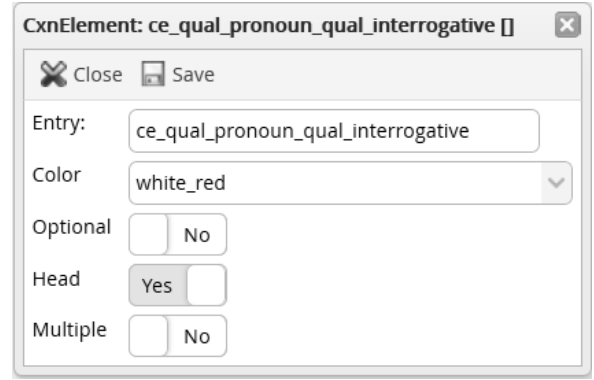

Figura 5. Edição da entrada do Elemento de Construção Pronome_qual [Marção 2018:83]

A Figura 5 apresenta a tela de edição do EC Pronome_qual. Nela insere-se o nome interlingual do EC, o qual é composto pela sigla "ce" em inglês para Elemento de Construção, "qual_pronoun" refere-se ao pronome qual, a parte "qual_interrogative" se refere à construção a qual esse EC pertence. Em seguida é possível escolher a cor em que esse elemento aparecerá, se este é um elemento opcional ou não. Também é possível assinalar se esse EC é "head" ou não, isto é, se é nuclear ou não, no caso, marcamos como nuclear. Por fim, assinalamos que o EC não é múltiplo, pois não há a possibilidade de mais de um pronome QU ser conectado recursivamente nessa construção sem que ocorra uma coordenação. Esse passo também é salvo e uma nova tela aparece (Figura 6).

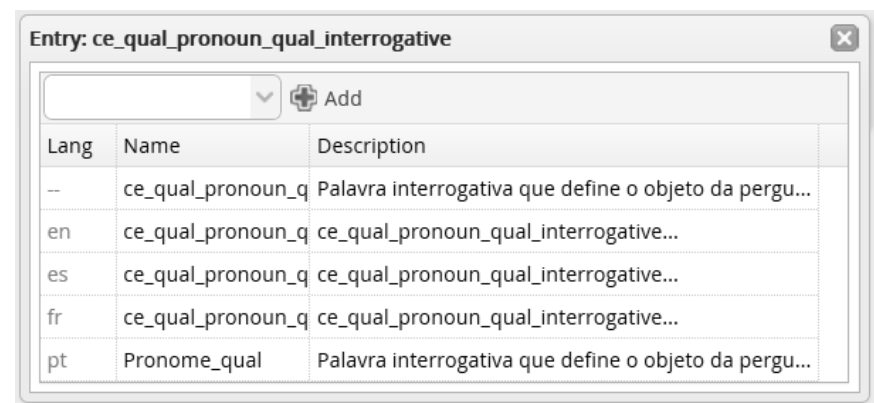

Figura 6. Edição entrada por idioma do Elemento de Construção Pronome_qual [Marção 2018:84]

A Figura 6 mostra a possibilidade de edição do EC por idioma. Clicando em "pt" abre-se uma nova janela, a qual é exibida na Figura 7. Nessa janela mantem-se o nome e cria-se a definição do EC em Português Brasileiro, contudo, em "Nick" mantemos o nome em inglês.

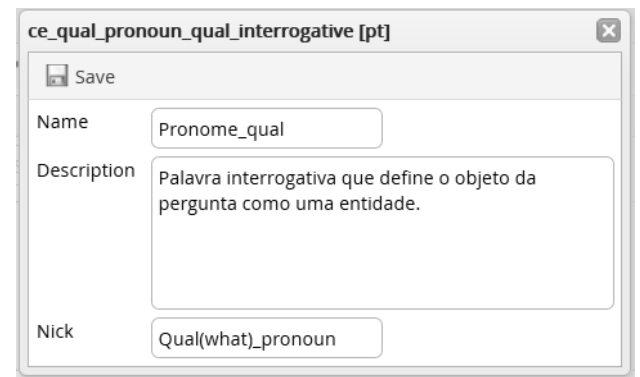

Figura 7. Edição da definição do Elemento de Construção Pronome_qual em Português Brasileiro [Marção 2018:84] 
Os mesmos procedimentos são realizados para o cadastramento do EC Estrutura_argumental_base, outro EC que compõe a construção Interrogativa_qual.

Depois da atribuição dos ECs, Marção (2018) estabelece as restrições de constituência da construção. Como vimos na seção 3, a constituência tipifica os ECs da construção em termos de outras construções. Assim, podemos assinalar se um EC é composto por uma outra construção já definida no Constructicon. Os ECs da construção Interrogativa_qual possuem essa restrição, o EC Estrutura_argumenta_base é licenciado pela construção Sujeito_predicado e o EC Pronome_qual é licenciado pela construção Pronome_QU, além disso, a restrição Lexeme (lex) especifica que é o pronome "qual" que deve ocupar a posição do pronome, como podemos ver na Figura 8.

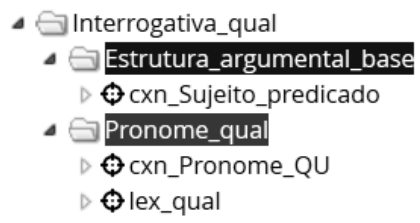

Figura 8. Restrições aplicadas à construção Interrogativa_qual [Marção 2018:85]

Por fim, a construção cadastrada é apresentada no Constructicon na forma da tela da Figura 9.

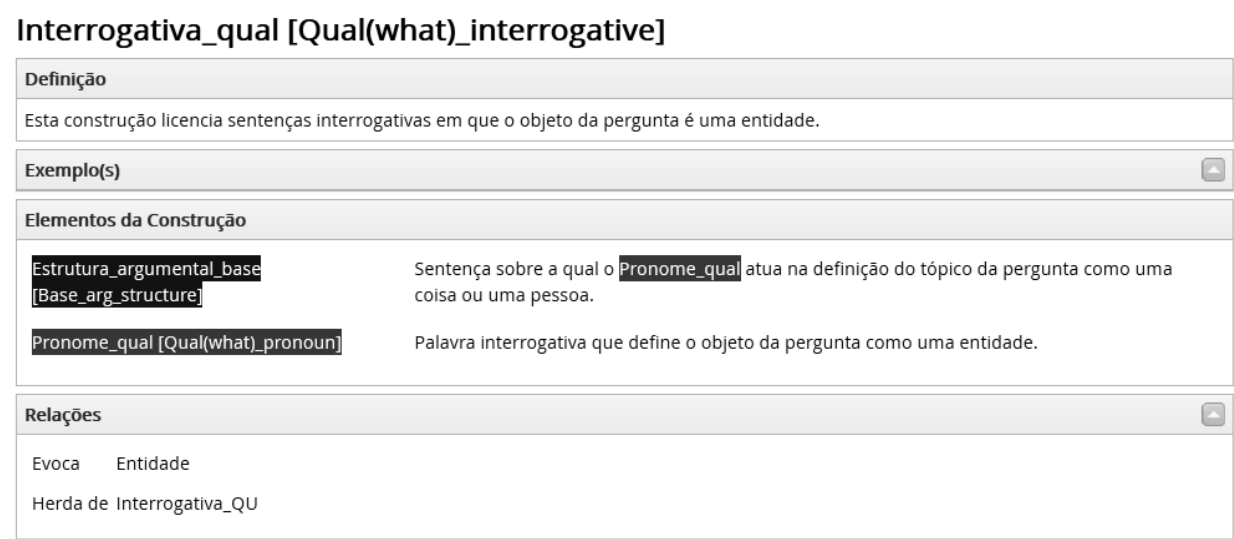

Figura 9. Construção Interrogativa_qual

Na Figura 9 vemos o nome da construção, seguido pelo nome interlingual. Abaixo temos a definição da construção. Em seguida, observamos os ECs Estrutura_argumental_base e Pronome_qual e suas definiçõos. Por fim, vemos que essa construção se relaciona com o Frame Entidade através da relação de Evocação e se relaciona a outra construção mais abstrata, Interrogativa_QU, através da relação de Herança.

O mesmo procedimento de modelagem se seguiu para as construções Interrogativa_que, Interrogativa_quem, Interrogativa_quando, Interrogativa_quanto, Interrogativa_onde, Interrogativa_como, Interrogativa_QU_preposicionada_razão e Interrogativa_QU_preposicionada_custo, respeitando as especificidades de cada construção [Marção 2018].

A partir da modelagem realizada para o total de onze construções, Marção (2018) propõe a rede construcional destas na Figura 10. 


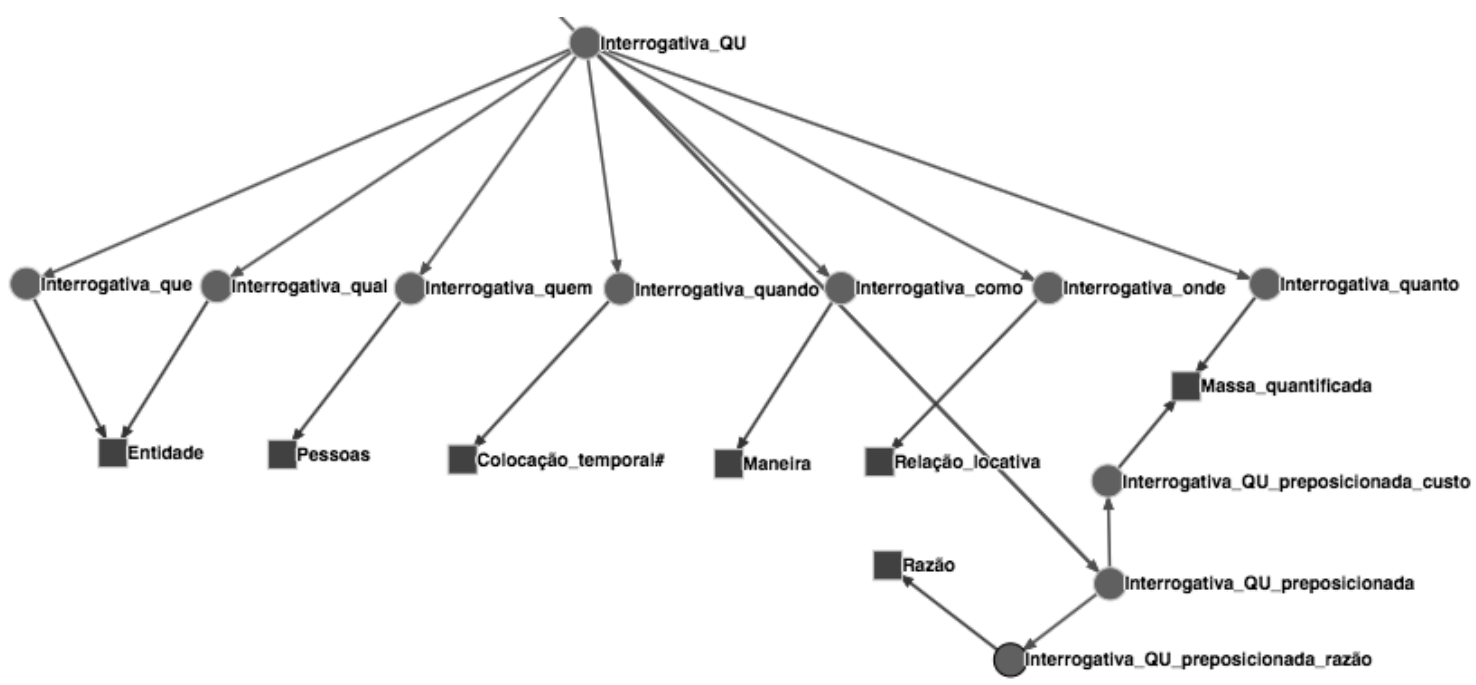

Figura 10. Rede de construções Interrogativas QU modeladas no Constructicon [Marção 2018:101]

A Figura 10 traz o gráfico que mostra as construções modeladas sob as relações de Herança e Evocação. Sendo assim, temos mais acima a construção mais abstrata, a Interrogativa_QU, a qual é herdada pelas demais construções representadas pelos círculos, incluindo a construção Interrogativa_QU_preposicionada, apresentada na parte inferior direita da imagem, a qual também é mais abstrata. Os quadrados representam os frames evocados por essas construções e a relação de Evocação é representada pelas setas que tomam a direção das construções aos Frames.

\section{Considerações Finais}

Neste texto apresentamos como a modelagem de construções se dá no Constructicon da FN-Br com base nos estudos de Marção (2018), demonstrando como a integração de aspectos da BCG e da Semântica de Frames enriquecem a representação formal e semântica das sentenças. Para isso, foram modeladas onze construções Interrogativas QU-, sendo a construção Interrogativa_QU mais abstrata e herdada pelas construções: Interrogativa_que; Interrogativa_qual; Interrogativa_quem; Interrogativa_quando; Interrogativa_quanto; Interrogativa_onde e Interrogativa_como. Também modelamos a Construção Interrogativa_QU_preposicionada mais abstrata, a qual é herdada pelas construções Interrogativa_QU_preposicionada_razão Interrogativa_QU_preposicionada_custo.

As construções cuja modelagem foi relatada neste trabalho poderão ser utilizadas em trabalhos futuros que tenham interesse, por exemplo, em analisar os tipos de estruturas argumentais que podem compor as construções Interrogativas QU-, identificar a relevância dessas estruturas para composição das construções Interrogativas QU- e que tipo de relação pode ser estabelecida entre as estruturas argumentais e o elemento QU, entre outras possibilidades de análise. 


\section{Referências}

Adger, D. (2003). Core syntax: A minimalist approach (Vol. 20). Oxford: Oxford University Press.

Augusto, M. R. (2005). QU deslocado e QU in situ no PB: aspectos da derivação lingüística e questões para a aquisição da linguagem. In IV Congresso Internacional da ABRALIN. Brasília: ABRALIN, p. 535-542.

Fillmore, C. J. (1985). Syntactic intrusions and the notion of grammatical construction. In Proceedings of the Annual Meeting of the Berkeley Linguistics Society. Berkeley, California: UC Berkeley, p. 73-86.

Fillmore, C. J. (2008). Border conflicts: FrameNet meets construction grammar. In Proceedings of the XIII EURALEX international congress. Barcelona, Spain: IULA., p. 49-68.

Fillmore, C. J., Lee-Goldman, R., \& Rhomieux, R. (2012). The FrameNet Constructicon. In Boas, H. C.; Sag, I. A. Sign-Based Construction Grammar. Stanford: CSLI, p. 309-372.

Fillmore, C. J. (2013). Berkeley Construction Grammar. In: Hoffmann, T.; Trousdale, G. (eds.). The Oxford Handbook of Construction Grammar. Oxford: Oxford University Press, p. 111-132.

Fried, M., \& Östman, J. O. (2004). Construction Grammar in a cross-language perspective. Amsterdam: John Benjamins.

Goldberg, A. (2006). Constructions at Work: The nature of generalization in language. Oxford: Oxford University Press.

Kay, P., and Fillmore, C. J. (1999). Grammatical constructions and linguistic generalizations: the What's X doing Y? construction. Language, 75(1), p. 1-33.

Marção, N. D. (2018). As construções interrogativas QU- no Constructicon da FrameNet Brasil. Dissertação de Mestrado em Linguística - Universidade Federal de Juiz de Fora.

Modesto, M. (2012). O programa minimalista em sua primeira versão. In Figueiredo de Alencar., L.; Othero, G. de A. (eds). Abordagens computatcionais da Teoria da Gramática. São Paulo: Mercado de Letras, p. 127-152.

Sag, I. A. (2012). Sign-Based Construction Grammar: An informal synopsis. In Boas, H. C.; Sag, I. A. Sign-Based Construction Grammar. Stanford: CSLI, p. 69-202.

Torrent, T. T., Matos, E., Lage, L., Laviola, A., Tavares, T., Almeida, V. G., \& Sigiliano, N. (2018). Towards continuity between the lexicon and the constructicon in FrameNet Brasil. In Lyngfelt, B., Borin, L., Ohara, K. \& Torrent, T. T. (eds.). Constructicography: Constructicon development across languages. Amsterdam: John Benjamins, p. 107-141. 